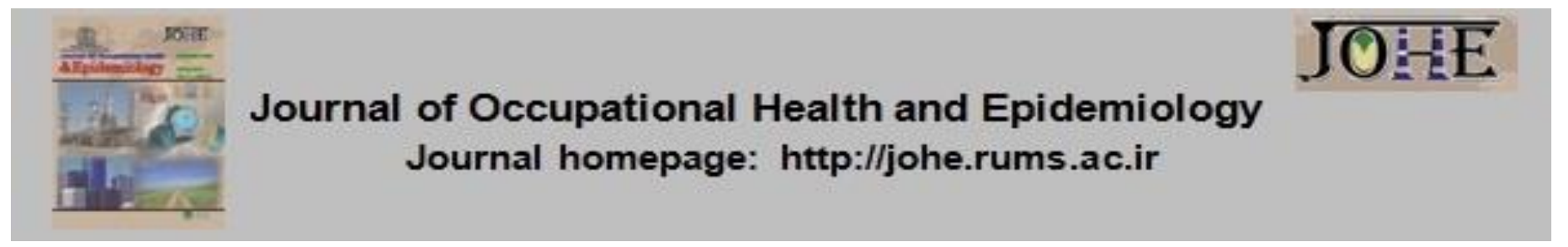

\title{
Malaria Endemicity Effect on Incidence and Mortality Rate of COVID-19 in Some Malaria-Endemic Regions of Iran; An Ecological Study (2020-21)
}

\author{
Golsoom Rashid ${ }^{1}$, Faeghe Zareei², Shokrollah Mohseniं ${ }^{3}$, Abdolhossein Madani ${ }^{4}$, Moussa Soleimani- \\ Ahmadi $^{5}$, Zahra Alebrahim 6 , Alireza Sanei-Dehkordi ${ }^{*}$
}

1. Msc in Parasitology, Social Determinants in Health Promotion Research Center, Hormozgan Health Institute, Hormozgan University of Medical Sciences, Bandar Abbas, Iran.

2. Msc in Microbiology, Social Determinants in Health Promotion Research Center, Hormozgan Health Institute, Hormozgan University of Medical Sciences, Bandar Abbas, Iran.

3. PhD Student in Epidemiology, Social Determinants in Health Promotion Research Center, Hormozgan Health Institute, Hormozgan University of Medical Sciences, Bandar Abbas, Iran.

4. Associate Prof., Social Determinants in Health Promotion Research Center, Hormozgan Health Institute, Hormozgan University of Medical Sciences, Bandar Abbas, Iran.

5. Professor, Dept. of Medical Entomology and Vector Control, School of Health, Hormozgan University of Medical Sciences, Bandar Abbas, Iran.

6. Assistant Prof., Social Determinants in Health Promotion Research Center, Hormozgan Health Institute, Hormozgan University of Medical Sciences, Bandar Abbas, Iran.

7. Associate Prof., Dept. of Medical Entomology and Vector Control, School of Health, Hormozgan University of Medical Sciences, Bandar Abbas, Iran.

\section{Citation: Rashid G, Zareei F, Mohseni S, Madani A, Soleimani-Ahmadi M, Alebrahim Z, CrossMark Sanei-Dehkordi A. Malaria Endemicity Effect on Incidence and Mortality Rate of COVID-19 in Some Malaria-Endemic, Regions of Iran; An Ecological Study (2020-21). J Occu Health Epidemiol 2021; 10(3):169-74.}

\section{Article Info}

* Corresponding author:

Alireza Sanei-Dehkordi,

E-mail:

Alireza.sanee@gmail.com

\section{Article history}

Received: Sep 2021

Accepted: Nov 2021

10.52547/johe.10.3.169

Print ISSN: 2251-8096 Online ISSN: 2252-0902

Peer review under responsibility of Journal of Occupational Health and Epidemiology

\section{Abstract}

Background: According to recent reports during the COVID-19 pandemic, malariaendemic regions have a significantly lower COVID-19 case and mortality rate than those malaria-non-endemic. This study aimed to evaluate the prevalence of COVID-19 in malarious areas of southern Iran and the incidence of disease in people previously infected with malaria.

Materials and Methods: We conducted an ecological study during the Covid-19 epidemic period in a malaria-endemic area, Hormozgan province, by including all critically ill patients with Covid-19 admitted to hospitals in different counties. The malaria cases were matched in the Covid patient's list. Also, the cumulative incidence and mortality rate of the Covid-19 were calculated in all counties.

Results: The results show that malaria-endemic regions have a lower cumulative incidence and mortality rate than the others. Moreover, it was found that people with a history of malaria were not infected with Covid 19.

Conclusion: It seems that malaria endemicity may positively affect the lower incidence and mortality rate of COVID-19 in malaria-endemic regions. However, further studies must be carried out to confirm the hypothesis that malaria reduces the risk of Covid-19 in other parts of the world.

Keywords: COVID-19, Malaria, Iran.

\section{Introduction}

Coronavirus disease 2019 (COVID-19) is an infectious respiratory illness caused by the severe acute respiratory syndrome coronavirus 2 (SARSCoV-2). The outbreak started in China in December 2019. It was declared a Public Health Emergency of International Concern (PHEIC) by 
the World Health Organization (WHO) in January 2020, and then a pandemic in March 2020 [1].

During the pandemic, malaria-endemic regions have a significantly lower COVID-19 case and mortality rate than other regions. Available statistics show a strong negative correlation between SARS-CoV-2 and malaria endemicity [2]. According to a malaria report in 2020, Nigeria (27\%), the Democratic Republic of the Congo $(12 \%)$, Uganda (5\%), Mozambique (4\%), Niger (3\%), Burkina Faso (3\%), Côte d' Ivoire (3\%), Angola (3\%), Mali (3\%), United Republic of Tanzania (3\%), and Cameroon (3\%) have accounted for about $69 \%$ of all malaria cases globally [3].

The remarkable point is that the number of confirmed cases and mortality rates of COVID-19 is relatively low in these malarious countries, including Nigeria (151000 (0.072\%) confirmed cases and 1800 deaths), the Democratic Republic of the Congo (23700 (0.023\%) confirmed cases and 680 deaths), Uganda (40100(0.086) confirmed cases and 330 deaths), Mozambique (54000 $(0.169 \%)$ confirmed cases and 580 deaths), Niger (4700 $(0.019 \%)$ confirmed cases and 170 deaths) Burkina Faso (11700 (0.055\%) confirmed cases and 130 deaths), Côte d' Ivoire (31000 (0.11\%) confirmed cases and 185 deaths), Angola (20400 $(0.06 \%)$ confirmed cases and 490 deaths), Mali (8200 (0.04\%) confirmed cases and 340 deaths), the United Republic of Tanzania (500 (0.0008\%) confirmed cases and 20 deaths), Mali (8250 $(0.04 \%)$ confirmed cases and 340 deaths), and Cameroon (33700 (0.12\%) confirmed cases and 520 deaths) $[4,5]$.

The total number of people in these countries is approximately 500 million, with 379000 confirmed COVID1-19 cases and 5200 deaths. In these African malarious countries, fewer than ten deaths/millions of the population were reported on average, while the world average mortality of the disease is about 103 people per million $[4,5]$.

According to previous studies, the angiotensinconverting enzyme gene (ACEI/D) and the angiotensin-converting enzyme 2 (ACE2) polymorphisms could partly explain this variable prevalence [6]. Anti- Glucose-6-Phosphate Isomerase (GPI) antibodies that develop in malaria patients could be identified as SARS-CoV-2 glycoproteins and play a protective role against COVID-19 [7]. Also, there are many theories on the prophylaxis effect of some antimalarial drugs, such as chloroquine (CQ) and hydroxychloroquine (HCQ). $\mathrm{HCQ}$ and $\mathrm{CQ}$ target $\mathrm{pH}$-dependent internalization and fusion by increasing lysosomes $[8,9]$.

Iran is one of the malaria-endemic countries in the world. According to the Ministry of Health, the most important malaria foci are located in Sistan \& Baluchistan, Hormozgan, and Kerman provinces in the south and southeastern parts of Iran, with more than four-fifths of cases [10]. Iran is currently at an advanced phase of malaria elimination; however, few indigenous cases of the disease are reported every year [11]. It reported zero indigenous malaria cases for the first time in 2018 [12]. Hormozgan province is one of the most important and oldest malaria-endemic areas in the south. The incidence of malaria has significantly reduced during the past 2 decades in this province [13,14]; however, some of its counties are still considered central endemic areas of malaria (Fig. 1).

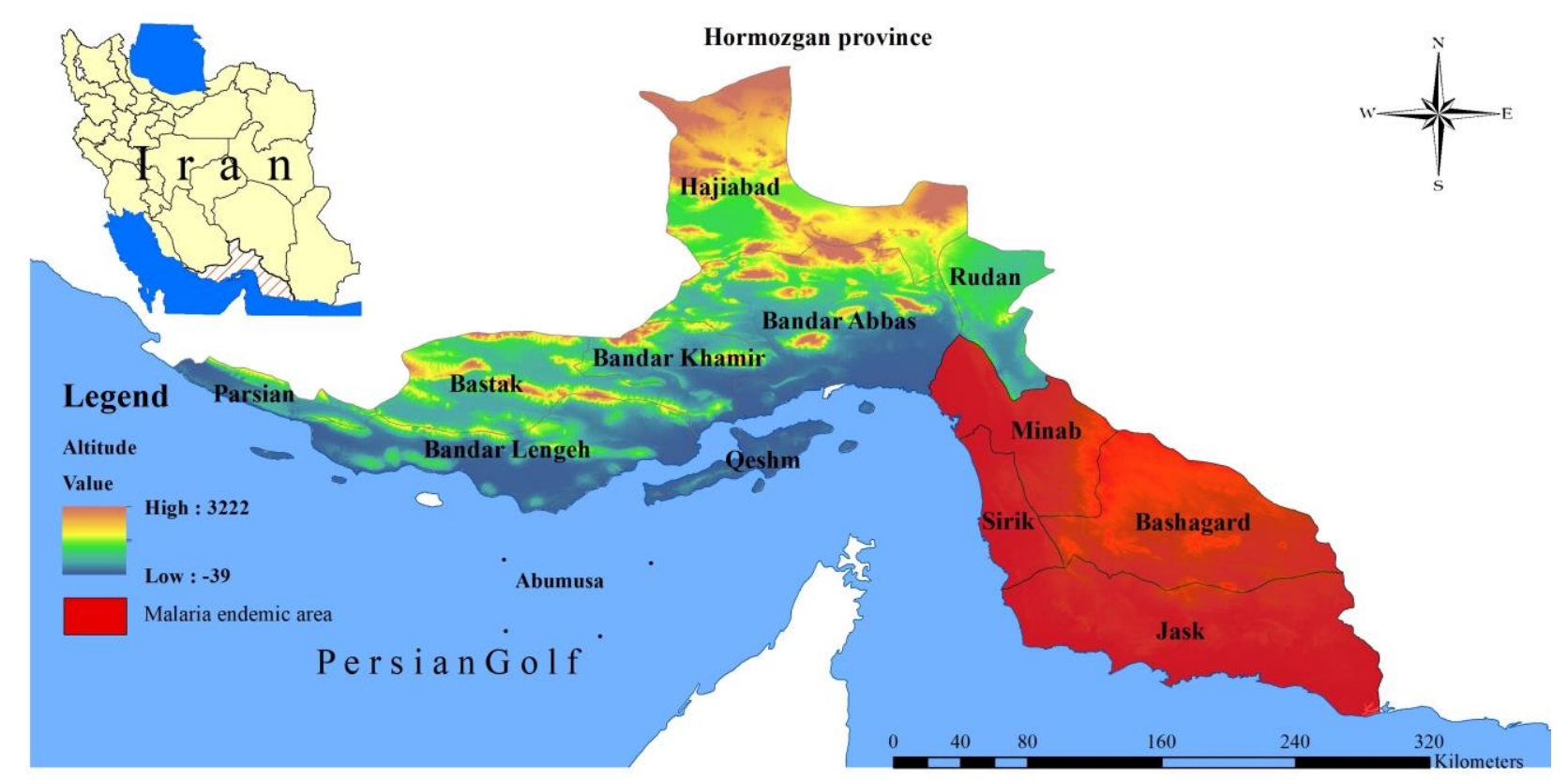

Fig. 1. Map of the Hormozgan province, highlighting the malaria-endemic areas [11] 
This study aimed to evaluate the prevalence of COVID-19 in malarious areas of southern Iran and the incidence of the disease in people previously infected with malaria.

\section{Materials and Methods}

We conducted an ecological study during the Covid-19 epidemic period in Hormozgan province from February of 2020 to February of 2021 by including all critically ill patients with Covid-19 admitted to hospitals in different counties. These patients were diagnosed according to the clinical guideline for COVID-19 diagnosis and molecular tests. Hormozgan province is a malaria-endemic area located in the south of Iran and north of the Persian Gulf between the geographical coordinates of $25.4^{\circ}-28.95^{\circ} \mathrm{N}$ and $53.68^{\circ}-59.25^{\circ}$ E. Approximately $38 \%$ of all malaria cases of Iran have occurred in this province [15]. Currently, malaria-endemic areas in this province are Bashagard, Jask, Minab, and Sirik counties (Fig. 2).

Demographic information of COVID-19 patients of all counties, including full name, national number, age, sex, occupation, and living areas, was recorded in a particular form. Non-indigenous people and those with less than ten years of stay in Hormozgan were excluded from the study. All patients were interviewed for the history of malaria in previous years. Also, the cumulative incidence and mortality rate of the Covid-19 were calculated in counties.

On the other hand, the number of malaria cases in each county and personal details of malaria patients over the past ten years in Hormozgan province were received from the Hormozgan Health Center. The malaria cases were searched and matched by full name, national number, and father's name in the Covid patient's list of Hormozgan province.

Related maps were prepared using Arc-GIS software, version 10.6 (Redlands, CA).

This study was approved by the Ethics Committee of the Hormozgan University of Medical Sciences (Ref. no: IR.HUMS.REC.1399.072).

\section{Results}

During the study period, 2020-2021, 38637 COVID-19 cases were reported in Hormozgan province by the Hormozgan Health Center (Table $1)$. The sex ratio was determined as $1.5(\mathrm{M} / \mathrm{F})$.

The age of patients ranged from 12 days to 109 years. Age groups were: $1 \%$ between $0-10$ years, $5 \%$ between $11-20$ years, $21 \%$ between $21-30$ months, $33 \% 31-40$ years, $18 \%$ between $41-50$ months, 33\% between $31-40$ years, $18 \%$ between 41-50 months, $10 \%$ between $51-60$ years, $7 \%$ between $61-70$ years, $3 \%$ between $71-80$ years, $1 \%$ between $81-90$ months, and $0.3 \%$ was 91 years and higher. The majority of the patients were housewives (27.1\%), service personnel (22.66), employees (20.84), workers (6.65), militaries (6.04), healthcare personnel (5.34), students (4.68), retirees (3.21), engineers (0.62), respectively.

Table 1. Information about Covid-19 and Malaria in different counties of Hormozgan province

\begin{tabular}{|c|c|c|c|c|c|}
\hline Counties & $\begin{array}{l}\text { Cases of } \\
\text { Covid-19 }\end{array}$ & $\begin{array}{c}\text { Cumulative } \\
\text { incidence }{ }^{*} 10^{4}\end{array}$ & Mortality & $\begin{array}{l}\text { Mortality } \\
\text { rates }^{*} 10^{4}\end{array}$ & $\begin{array}{c}\text { Malaria cases } \\
(2010-2020)\end{array}$ \\
\hline Abumusa & 42 & 209.27 & 0 & 0 & 0 \\
\hline Bandar Abbas & 18868 & 295.44 & 486 & 7.61 & 20 \\
\hline Bandar Lengeh & 2124 & 174.65 & 82 & 6.74 & 7 \\
\hline Bandar Khamir & 1236 & 204.35 & 30 & 4.96 & 1 \\
\hline Bashagard & 550 & 182.58 & 1 & 0.33 & 25 \\
\hline Bastak & 1583 & 189.91 & 35 & 4.2 & 0 \\
\hline Gheshm & 3754 & 239.04 & 85 & 5.41 & 7 \\
\hline Hajiabad & 1697 & 282.61 & 19 & 3.16 & 0 \\
\hline Jask & 919 & 150.34 & 17 & 2.78 & 68 \\
\hline Minab & 3812 & 143.12 & 96 & 3.6 & 18 \\
\hline Parsian & 1096 & 200.33 & 24 & 4.39 & 0 \\
\hline Rudan & 2226 & 170.58 & 80 & 6.13 & 3 \\
\hline Sirik & 730 & 142.18 & 8 & 1.56 & 3 \\
\hline
\end{tabular}

The number of malaria cases between 2010 to 2020 was 151 in Hormozgan province (Table 1, Fig. 4). The distribution pattern of malaria cases was consistent with its endemicity in this province, so that Jask county and Bashagard county had the highest number of malaria cases. After matching, it was found that people with a history of malaria were not infected with Covid 19. 


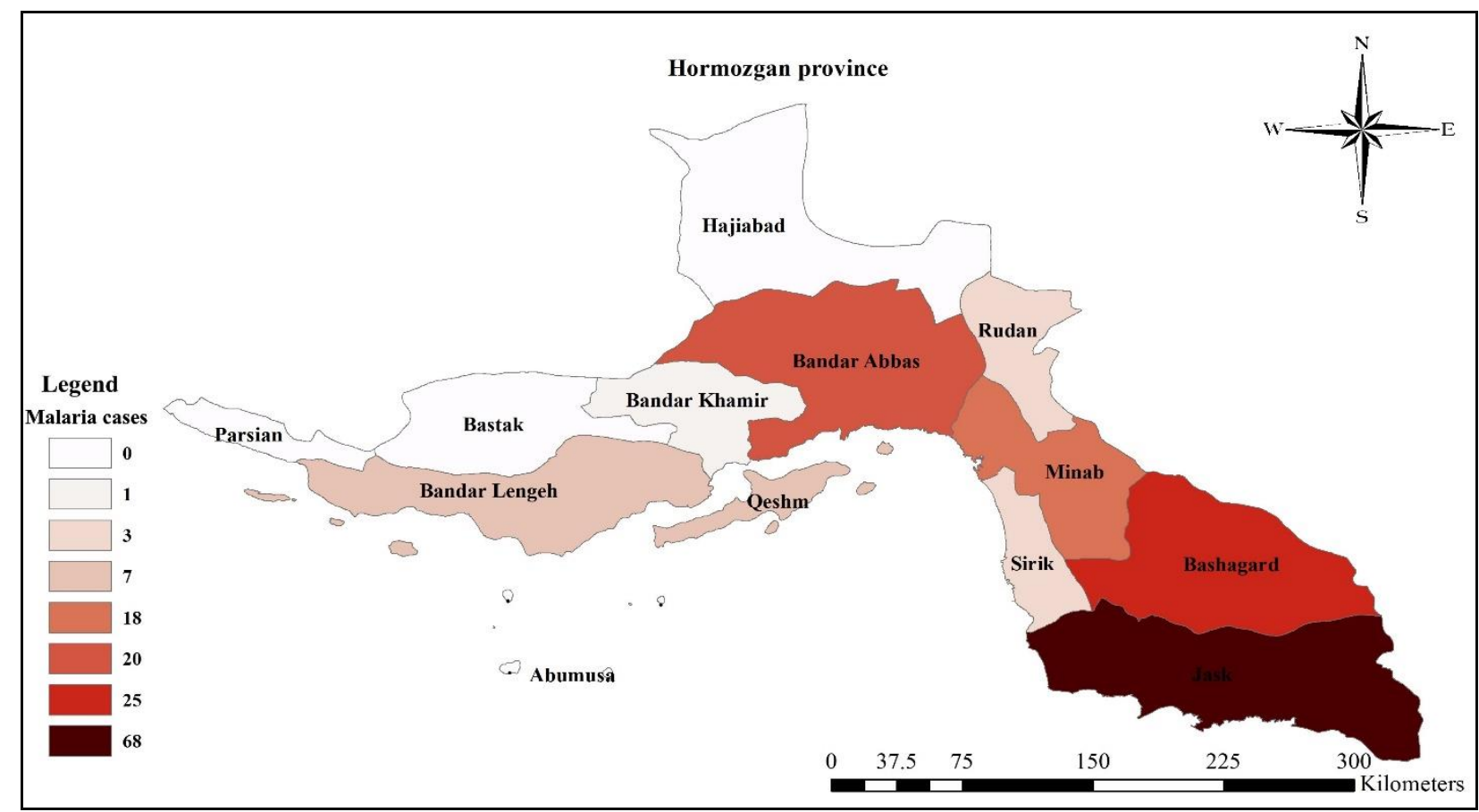

Fig.4. The number of malaria cases in Hormozgan province from 2010 to 2020

The cumulative incidence and mortality rate of COVID-19 show that the eastern areas of the province, which are malaria-endemic regions, have a lower incidence of Covid 19 (Table1, Fig. 5).

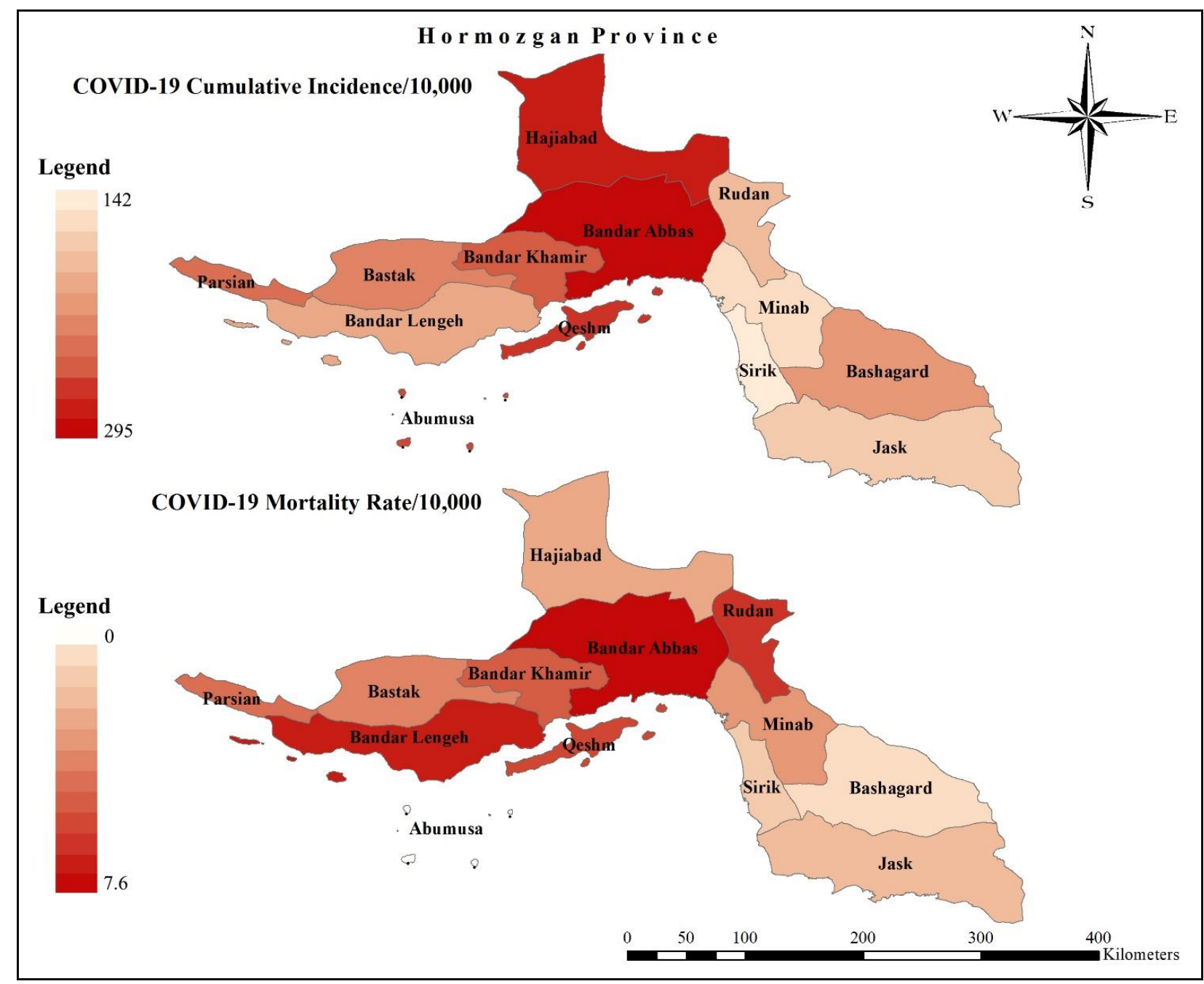

Fig. 5. The cumulative incidence and mortality rate of COVID-19 in Hormozgan province from 2020 to 2021 


\section{Discussion}

The results show that some malaria-endemic areas of Iran have a lower incidence and mortality rate of Covid-19 than others. Also, based on our findings, none of the people with a history of malaria have been diagnosed with COVID-19. However, the number of 10-year malaria cases in Hormozgan province was very low since Iran is moving toward a malaria eradication program, and with this number of malaria cases, it is not possible to make a correct judgment about the relationship between the history of malaria and Covid-19. These findings are in line with previous studies, based on which malaria can reduce the incidence of Covid 19 through various mechanisms. The main factors to justify this hypothesis are ACEI/D, ACE2, Anti- GPI antibodies in malaria patients, and the prophylaxis effect of some antimalarial drugs [6-9]. Lestari et al. (2020) show that some antimalarial drugs, such as quinine, chloroquine, and hydroxychloroquine, can be used as a COVID-19 antidote since they block the infection of the SARS-CoV-2 virus by interacting with residue Lys353 in the peptidase domain of the ACE2 receptor [16]. Artemisininpiperaquine is currently used as an effective drug in treating cerebral and chloroquine-resistant falciparum malaria. Li et al. (2021) indicate that in patients with COVID-19, the time to reach undetectable SARS-CoV-2 is significantly shorter in the artemisinin-piperaquine group than in the control group [17]. Further studies are needed to explore the exact role of malaria endemicity on the incidence of COVID-19.

\section{Conclusion}

It seems that malaria endemicity positively affects the lower incidence and mortality rate of COVID-19 in malaria-endemic regions. However, further studies must be conducted to confirm the hypothesis that malaria reduces the risk of Covid19 in other parts of the world, especially in African countries where malaria is endemic.

\section{Acknowledgement}

The authors would like to thank Dr. Hossein pour, Dr. Fakhar, and Eng. Fekri in Hormozgan Health Center for their assistance in gathering the necessary data for this work. This study received financial support from the Research Deputy of Hormozgan University of Medical Sciences (Project No. 99084).

Conflict of interest: None declared.

\section{References}

1. World Health Organization. Coronavirus disease 2019 (COVID-19): situation report, 82. Geneva, Switzerland: World Health Organization; 2020.

2. Lawal Y. Africa's low COVID-19 mortality rate: $A$ paradox? Int J Infect Dis 2021; 102:118-22.

3. World Health Organization. World Malaria Report 2020: Geneva, Switzerland: World Health Organization; 2020.

4. Worldometers. COVID-19 Coronavirus Pandemic: Reported Cases and Deaths by Country. 2021 Feb 20. Available from: https://www.worldometers.info/coronavirus/\#cou ntries

5. Worldometers. World Population by Country. 2021 Feb 20. Available from: https://www.worldometers.info/worldpopulation/population-by-country/

6. Bosso M, Thanaraj TA, Abu-Farha M, Alanbaei M, Abubaker J, Al-Mulla F. The Two Faces of ACE2: The Role of ACE2 Receptor and Its Polymorphisms in Hypertension and COVID-19. Mol Ther Methods Clin Dev 2020; 18:321-7.

7. Parodi A, Cozzani E. Coronavirus disease 2019 (COVID 19) and Malaria. Have anti glycoprotein antibodies a role? Med Hypotheses 2020; 143:110036.

8. Gao J, Tian Z, Yang X. Breakthrough: Chloroquine phosphate has shown apparent efficacy in treatment of COVID-19 associated pneumonia in clinical studies. Biosci Trends 2020; 14(1):72-3.

9. Shah S, Das S, Jain A, Misra DP, Negi VS. A systematic review of the prophylactic role of chloroquine and hydroxychloroquine in coronavirus disease-19 (COVID-19). Int $\mathrm{J}$ Rheum Dis 2020; 23(5):613-9.

10. Raeisi A, Gouya MM, Nadim A, Ranjbar M, Hasanzehi A, Fallahnezhad $M$, et al. Determination of Malaria Epidemiological Status in Iran's Malarious Areas as Baseline Information for Implementation of Malaria Elimination Program in Iran. Iran J Public Health 2013; 42(3):326-33.

11. Vatandoost $H$, Raeisi $A$, Saghafipour $A$, Nikpour F, Nejati J. Malaria situation in Iran: 2002-2017. Malar J 2019; 18(1):200.

12. Azizi $H$, Davtalab-Esmaeili E, Farahbakhsh $M$, Zeinolabedini M, Mirzaei Y, Mirzapour M. Malaria situation in a clear area of Iran: an approach for the better understanding of the health service providers' readiness and challenges for malaria elimination in clear areas. Malar J 2020; 19(1):114.

13. Sanei-Dehkordi A, Soleimani-Ahmadi M, Jaberhashemi SA, Zare M. Species composition, seasonal abundance and distribution of potential anopheline vectors in a malaria endemic area of Iran: field assessment for malaria elimination. Malar J 2019; 18(1):157.

14. Zare M, Soleimani-Ahmadi M, Davoodi SH, Sanei-Dehkordi A. Insecticide susceptibility of 
Anopheles stephensi to DDT and current insecticides in an elimination area in Iran. Parasit Vectors 2016; 9(1):571.

15. Ghahremani L, Azizi M, Moemenbellah-Fard MD, Ghaem H. Malaria preventive behaviors among housewives in suburbs of Bandar-Abbas City, south of Iran: interventional design based on PRECEDE model. Pathog Glob Health 2019; 113(1):32-8.

16. Lestari K, Sitorus T, Instiaty, Megantara S, Levita J. Molecular Docking of Quinine,
Chloroquine and Hydroxychloroquine to Angiotensin Converting Enzyme 2 (ACE2) Receptor for Discovering New Potential COVID19 Antidote. J Adv Pharm Educ Res 2020; 10(2):1-4.

17. Li G, Yuan M, Li H, Deng C, Wang Q, Tang Y, et al. Safety and efficacy of artemisininpiperaquine for treatment of COVID-19: an open-label, non-randomised and controlled trial. Int J Antimicrob Agents 2021; 57(1):106216. 